\title{
Quantitative assessment of carotid artery atherosclerosis by three-dimensional magnetic resonance and two-dimensional ultrasound imaging: a comparison study
}

\author{
Huiyu Qiao', Ying Cai ${ }^{2}$, Manwei Huang ${ }^{3}$, Yang Liu ${ }^{4}$, Qiang Zhang ${ }^{1}$, Lingyun Huang ${ }^{5}$, Huijun Chen ${ }^{1}$, \\ Chun Yuan', Xihai Zhao ${ }^{1}$ \\ ${ }^{1}$ Center for Biomedical Imaging Research, Department of Biomedical Engineering, Tsinghua University School of Medicine, Beijing 100084, China; \\ ${ }^{2}$ Department of Radiology, Taizhou People's Hospital, Taizhou 225400, China; ${ }^{3}$ Department of Ultrasound, China Meitan General Hospital, Beijing \\ 100028, China; ${ }^{4}$ Department of Radiology, The Affiliated Hospital of Yangzhou University, Yangzhou 225009, China; ${ }^{5}$ PingAn Technology, Shanghai \\ 200040, China; ${ }^{6}$ Department of Radiology, University of Washington, Washington, Seattle, USA
}

Correspondence to: Xihai Zhao, MD, PhD. Center for Biomedical Imaging Research, Tsinghua University School of Medicine, Haidian District, Beijing 100084, China. Email: xihaizhao@tsinghua.edu.cn.

Background: It has been proven that magnetic resonance (MR) and ultrasound imaging are useful tools in the quantification of carotid atherosclerotic plaques. However, there are only a few pieces of evidence to illustrate the links of quantitative measurements of carotid plaques between MR and ultrasound imaging. This study looked to compare the quantitative measurements of carotid plaques and investigate their relationship between three-dimensional (3D) MR vessel wall imaging and two-dimensional (2D) ultrasound imaging.

Methods: Seventy-five asymptomatic elderly subjects (mean age: $73.3 \pm 5.7$ years; 45 males) with carotid atherosclerotic plaques diagnosed by both ultrasound and MR imaging were included in this study. The plaque size, including the maximum wall thickness (Max WT), plaque length, and plaque area, was measured by $3 \mathrm{D} \mathrm{MR}$ and ultrasound imaging on longitudinal and cross-sectional views. The quantitative assessments of carotid plaque size were compared and correlated between 3D MR and 2D ultrasound imaging.

Results: In total, the quantitative measurements of 101 plaques on longitudinal views or 44 plaques on cross-sectional views of both MR and ultrasound imaging were compared. The Max WT of the plaques (longitudinal: $2.9 \pm 0.8$ vs. $2.4 \pm 0.9 \mathrm{~mm}$; cross-sectional: $3.2 \pm 1.1$ vs. $2.6 \pm 0.7 \mathrm{~mm}$ ) and plaque areas (longitudinal: $24.3 \pm 13.4$ vs. $17.0 \pm 12.7 \mathrm{~mm}^{2}$; cross-sectional: $24.9 \pm 24.6$ vs. $16.8 \pm 13.3 \mathrm{~mm}^{2}$ ) measured by MR imaging were found to be significantly higher than those measured by ultrasound imaging (all $\mathrm{P}<0.001$ ). Moderate to strong correlations were found in Max WT, plaque area, plaque length between 3D MR and ultrasound imaging.

Conclusions: The quantitative measurements of carotid plaques using 3D MR and 2D ultrasound are significantly correlated. The plaque area and Max WT measured by 3D MR imaging are more significant than these parameters measured by 2D ultrasound imaging, which might be explained by the resolution of MR imaging and the workflow of measurements.

Keywords: Atherosclerosis; carotid arteries; magnetic resonance imaging; ultrasound imaging

Submitted Sep 30, 2019. Accepted for publication Apr 14, 2020.

doi: $10.21037 /$ qims-19-818

View this article at: http://dx.doi.org/10.21037/qims-19-818 


\section{Introduction}

Vulnerable atherosclerotic plaques in carotid arteries have been shown to be closely associated with ischemic cerebrovascular events, such as stroke or transient ischemic attack (1-3). Therefore, it is vital to assess the vulnerability of carotid plaques before such clinical events occur. Earlier studies reported that morphological measurements, including wall thickness and plaque area, were useful indicators for plaque vulnerability and vascular events (4-6). Hence, the accurate measurement of plaque size is valuable for disease stratification.

Two-dimensional (2D) ultrasound imaging is considered to be a suitable tool for screening carotid atherosclerosis due to its low cost and full availability. It is well established that the area and thickness of a plaque measured by ultrasound imaging serve as useful indicators of plaque progression (5) and cerebrovascular events $(7,8)$. However, the reliability of ultrasound imaging depends on the proficiency and experience of the operator, although automated edge detection algorithms have been developed to reduce the inter-operator variability of measurements (9).

Earlier studies showed that MR imaging possessed excellent interscan (10-12), interreader (13), and intrareader (14) reproducibility in quantitative measurements of carotid atherosclerotic plaques. It has been shown that maximum wall thickness (Max WT), which is measured by MR imaging, was related to cerebrovascular events (15). However, quantitative measurements by $2 \mathrm{D}$ traditional MR imaging might introduce overestimation due to low resolution, which might cause a partial volume effect. The recommended in-plane and through-plane resolutions for $2 \mathrm{D}$ traditional MR imaging were 0.63 and $2 \mathrm{~mm}$, respectively (16). Recently, three-dimensional (3D) MR imaging with more extensive longitudinal coverage and isotropic high spatial resolution has been proposed and used for assessing plaques (17-19). This imaging technique can minimize the partial volume effect and allow reconstruction with flexible orientation during post-processing, which may yield correct quantification of plaque size.

Different results can be achieved by measuring plaque size using ultrasound and MR imaging. It is vital to align the quantitative measurements of carotid plaques, including Max WT, plaque length, and plaque area derived from different imaging modalities, to stratify cardiovascular disease risk and devise treatment strategies. Duivenvoorden et al. proved that the measurements of mean wall area and mean wall thickness on MR and the measurements of mean wall area and intima-media thickness on ultrasound were in good agreement $(20,21)$. Although the comparison between mean wall thickness on MR and intima-media thickness on ultrasound has been performed $(20,22)$, the difference in measurements of plaque area on MR and ultrasound have seldom been explored. Furthermore, the quantitative measurements of carotid plaques were usually obtained from a crosssectional view on MR images, and a longitudinal view on ultrasound images and previous comparisons between MR and ultrasound were utilized on their dimension, which might not be comparable. There is less evidence to illustrate the links of quantitative measurements of carotid plaques between 3D MR and 2D ultrasound imaging on the same dimensions.

This study looked to compare the quantitative measurements of carotid plaques and investigate their relationship between $3 \mathrm{D}$ MR vessel wall imaging and 2D ultrasound imaging.

\section{Methods}

\section{Study sample}

All subjects were recruited from the community study "Cardiovascular Risk Of Elderly Population (CROP)." The purpose of the CROP study was to investigate the cardiovascular risk in elderly subjects ( $\geq 60$ years old) without cardiovascular symptoms in recent 6 months using ultrasound and MR imaging. The present study included subjects with carotid plaques diagnosed by both ultrasound and MR imaging from CROP datasets. Clinical information, including age, gender, blood pressure, hyperlipidemia, hypertension, smoke, diabetes, and cardiovascular disease, was collected. The study protocol was supported by the local institutional review board, and all participants completed a written informed consent form.

\section{Carotid ultrasound imaging}

B-mode and color Doppler ultrasound imaging were performed for bilateral carotid arteries on a Philips iU22 ultrasound system with the L9-3 linear array transducer. The thickness and area of distal common carotid plaques and proximal internal carotid plaques were measured from the longitudinal and cross-sectional views of the plaques. To avoid intra-sonographer variations, all carotid ultrasound imaging was conducted by the same sonographer. 


\section{Carotid MR imaging}

All carotid arteries were imaged on a 3.0 T MR scanner (Achieva TX, Philips Healthcare, The Netherlands) with a custom-designed 36-channel cerebrovascular coil within two weeks after ultrasound imaging. A 3D Motionsensitized driven-equilibrium prepared rapid gradient echo (MERGE) (17) sequence was acquired in coronal view for bilateral carotid arteries with the following parameters: turbo field echo (TFE), repetition time/echo time 9.0/4.2 ms, flip angle $6^{\circ}$, TFE factor 90 , field of view $250 \times 160 \times 40 \mathrm{~mm}^{3}$, and spatial resolution $0.8 \times 0.8 \times 0.8 \mathrm{~mm}^{3}$. The scan time of MERGE was 4 minutes.

\section{Image analysis}

The longitudinal and cross-sectional views of ultrasound imaging were reviewed by a sonographer with 25 years' experience in carotid ultrasound imaging, who was blinded to MR images. The Max WT, plaque length, and plaque area were measured manually on the frozen longitudinal and cross-sectional ultrasound images at the plane with a maximum thickness of carotid plaque in cardiac diastole. The plaque on ultrasound images was defined as a focal thickening $>1.5 \mathrm{~mm}$, according to Mannheim consensus (23), which is a widely used criterion in clinical settings. For carotid arteries with multiple plaques, the most severe plaque at each artery was chosen for analysis to minimize the probability of mismatch of the target plaques between MR and ultrasound imaging.

The 3D MR images were reconstructed to the longitudinal and cross-sectional views using a multiplanar reconstruction algorithm before analysis with a Philips MR workstation (Extended MR Workspace 2.6.3.1, Philips Medical System, Best, Netherlands) and 3D CASCADE software, respectively (24). The MR image quality was assessed with a 4 -point scale: $1=$ poor; $2=$ marginal; $3=$ good; and $4=$ excellent (25). Slices with image quality $<2$ were excluded. For carotid arteries with multiple plaques, the most severe plaque was measured. The longitudinal views of MR images were reviewed by two radiologists with more than 3 years' experience in cardiovascular MR imaging using an MR workstation. The radiologists were blinded to ultrasound imaging. The Max WT, plaque length, and plaque area were measured manually on the longitudinal views of carotid images for each plaque.

The 3D MR images were resliced cross-sectionally with
$2 \mathrm{~mm}$ slice thickness and reviewed by two radiologists with more than 3 years of experience in cardiovascular MR imaging using a custom-designed 3D CASCADE software (24). The radiologists were blinded to ultrasound images. The intra-observer and inter-observer interclass correlation coefficient (ICC) was 0.961 (95\% CI, $0.887-$ 0.992 ) and 0.925 (95\% CI, 0.626-0.985), respectively, in mean wall thickness measured by 3D MR imaging (24). The boundaries of the lumen and outer wall were outlined semi-automatically and adjusted on the cross-sectional view, which is perpendicular to the centerline of the carotid artery. The boundaries and auto-calculated wall thickness information of each cross-sectional image were recorded in files. Each file was uploaded to MATLAB to calculate plaque area, which was defined as the area of the region (marked by a straight line in Figure 1) from Max WT to a threshold of wall thickness including 1.5, 1.6, 1.7, 1.8, $1.9,2.0$, and $2.1 \mathrm{~mm}$. The plaque area was measured at the cross-sectional images with the Max WT of each plaque.

\section{Reproducibility study}

Ten carotid arteries from ten subjects were randomly selected for the reproducibility study. Two sonographers reviewed the frozen longitudinal and cross-sectional ultrasound images separately. Two radiologists interpreted the longitudinal MR images separately. One sonographer and one radiologist respectively reviewed these ultrasound images and MR images again with a one-year time interval to minimize the memory bias.

\section{Statistical analysis}

The continuous variables were described as mean \pm standard deviation. The Max WT, plaque length, and plaque area were compared between 3D MR and ultrasound images on the longitudinal and cross-sectional views using a Wilcoxon signed-rank test. Bland-Altman analysis was performed to determine the differences in Max WT, plaque length, and plaque area between the MR and ultrasound imaging. Spearman's correlation was conducted to evaluate the correlation of the Max WT, plaque length, and plaque area measurements between 3D MR and ultrasound imaging. The inter-observer and intra-observer agreement of quantitative measurements on MR and ultrasound images were analyzed by calculating ICC and corresponding $95 \%$ CI. All statistical analyses were performed using SPSS 16.0 (SSPS Inc., Chicago, IL, USA). 

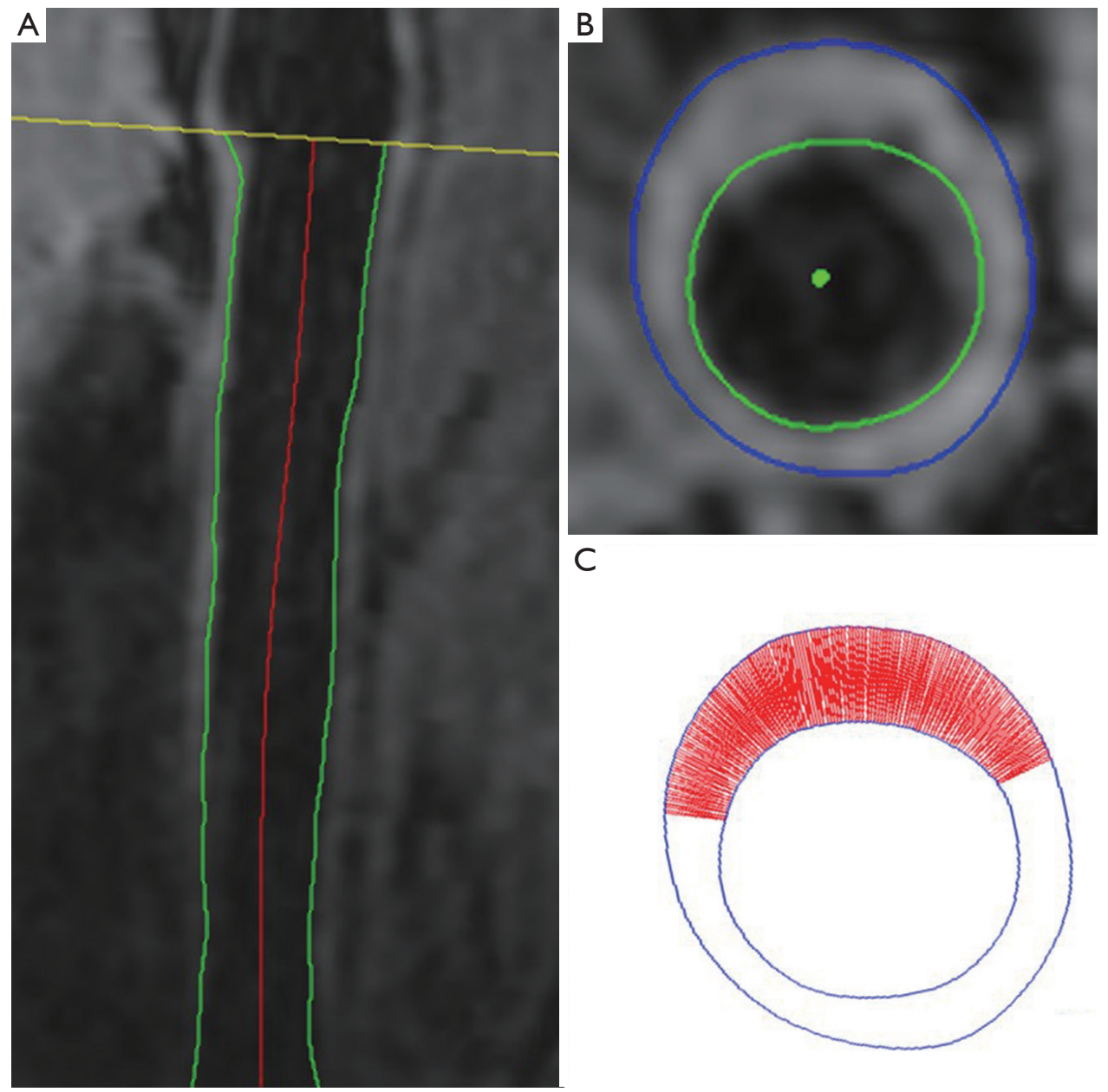

C

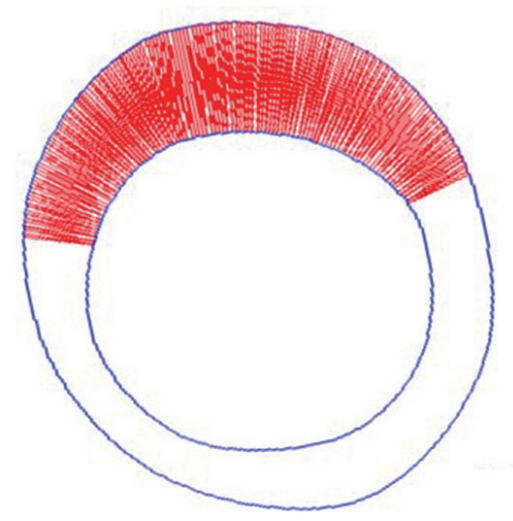

Figure 1 Methodology for measuring plaque area on MR images. The common carotid artery is shown in (A), and (B) is the cross-sectional slice with Max WT. The area marked by a straight line in (C) is the plaque area defined as the region from Max WT to threshold wall thickness (threshold wall thickness $=1.5 \mathrm{~mm}$ ).

\section{Results}

In the CROP study, 200 subjects were recruited, of which 146 subjects underwent both MR and ultrasound imaging. Of these 146 subjects, 6 subjects were excluded due to poor image quality. Of the remaining 140 subjects, 75 who had carotid atherosclerotic plaques diagnosed by both ultrasound and MR imaging were finally included in this study. In total, 75 subjects with acceptable image quality were included in the statistical analysis. The mean age of these 75 subjects was $73.3 \pm 5.7$ years old, 45 were males, 41 had hypertension, 12 had a history of smoking, 15 had diabetes, 57 had hyperlipidemia, and 13 had cardiovascular disease. The subjects' clinical information is detailed in Table 1. In the 75 subjects, 115 plaques were found to be eligible for quantification of plaque size on both MR and ultrasound imaging.

\section{Comparison of plaque measurements on longitudinal views between MR and ultrasound imaging}

In total, the measurements of 101 plaques with longitudinal views on both $M R$ and ultrasound imaging from 70 asymptomatic subjects were compared. The Max WT of plaque $(2.9 \pm 0.8$ vs. $2.4 \pm 0.9 \mathrm{~mm}, \mathrm{P}<0.001)$ and plaque area $\left(24.3 \pm 13.4\right.$ vs. $\left.17.0 \pm 12.7 \mathrm{~mm}^{2}, \mathrm{P}<0.001\right)$ measured by MR imaging were found to be significantly greater than those measured by ultrasound imaging. The carotid plaque area measured by MR imaging was approximately 1.43 fold greater than that measured by ultrasound imaging 
Table 1 Clinical information of study population ( $\mathrm{n}=75)$

\begin{tabular}{lcc}
\hline Variable & Mean \pm SD or n (\%) & Range \\
\hline Gender, male & $45(60.0)$ & - \\
Age, years & $73.3 \pm 5.7$ & $60-86$ \\
Body mass index, kg/m² & $23.8 \pm 3.0$ & $17.3-34.2$ \\
Smoke & $12(16.0)$ & - \\
Diabetes & $15(20.0)$ & - \\
Hypertension & $41(54.7)$ & - \\
Systolic blood pressure, & $133.5 \pm 15.2$ & $100-180$ \\
mmHg & & \\
Diastolic blood pressure, & $71.3 \pm 9.9$ & $49-100$ \\
mmHg & & - \\
Hyperlipidemia & $57(76.0)$ & - \\
Cardiovascular disease & $13(17.3)$ & \\
\hline
\end{tabular}

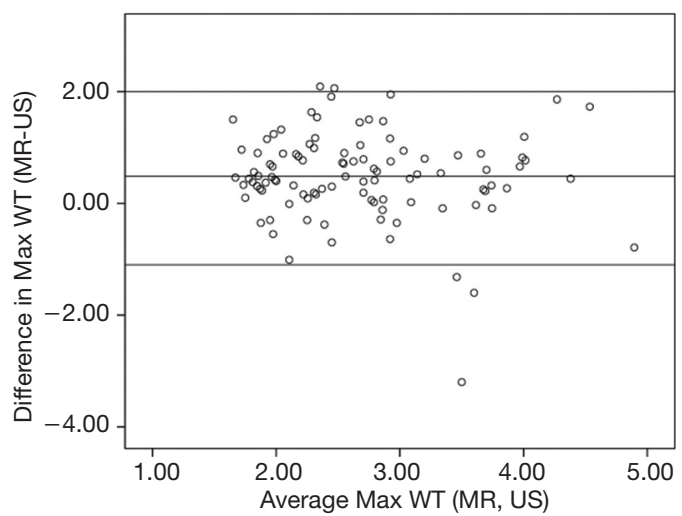

Figure 2 Bland-Altman analysis of Max WT measured by MR and ultrasound imaging on longitudinal views.

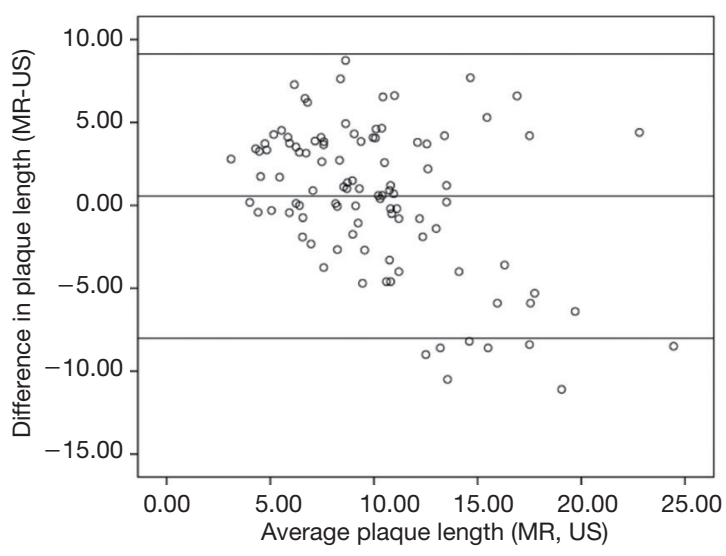

Figure 3 Bland-Altman analysis of plaque length measured by MR and ultrasound imaging on longitudinal views. on longitudinal views. Significant correlations were found in Max WT $(r=0.544, \mathrm{P}<0.001)$, plaque area $(\mathrm{r}=0.702$, $\mathrm{P}<0.001)$, and plaque length $(\mathrm{r}=0.597, \mathrm{P}<0.001)$ between $M R$ and ultrasound imaging. There was no significant difference in plaque length between MR and ultrasound imaging $(10.4 \pm 3.8$ vs. $9.8 \pm 5.5 \mathrm{~mm}, \mathrm{P}=0.062)$. BlandAltman analyses showed that Max WT, plaque length, and plaque area measured by MR on longitudinal views were significantly larger on average with limits of agreement of -1.1 to $2.0 \mathrm{~mm}$ (Figure 2), -8.0 to $9.1 \mathrm{~mm}$ (Figure 3), and -10.9 to $25.3 \mathrm{~mm}^{2}$ (Figure $4 A$ ), respectively.

\section{Comparison of plaque measurements on cross-sectional views between MR and ultrasound imaging}

In the CROP study, a cross-sectional ultrasound scan was not routine protocol and was only conducted for some of the subjects who were consecutively recruited in this study. Hence, the measurements of 44 plaques with crosssectional views on both $\mathrm{MR}$ and ultrasound imaging from 31 asymptomatic subjects were compared. The Max WT of plaque $(3.2 \pm 1.1$ vs. $2.6 \pm 0.7 \mathrm{~mm}, \mathrm{P}<0.001)$ measured by MR imaging were found to be significantly higher than that measured by ultrasound imaging. Significant correlation was found in Max WT $(r=0.692, \mathrm{P}<0.001)$ between MR and ultrasound imaging. The mean plaque area of all 44 plaques measured by ultrasound was $16.8 \pm 13.3 \mathrm{~mm}^{2}$. In contrast, the plaque area measured by MR imaging was significantly larger than that measured by ultrasound using a WT threshold from 1.5 to $2.1 \mathrm{~mm}$ (all $\mathrm{P}<0.001$, Table 2). When the threshold was set as $2.1 \mathrm{~mm}$ for wall thickness, the plaque area measured by MR imaging was 1.48-fold higher than that measured by ultrasound imaging on cross-sectional views. The correlation coefficients of the plaque area measured by MR and ultrasound increased from 0.603 to 0.712 with the WT threshold increased from 1.5 to $2.1 \mathrm{~mm}$ (all $\mathrm{P} \leq 0.001$, Table 2). Bland-Altman analyses showed that plaque area and Max WT measured by MR on crosssectional views were significantly larger on average with limits of agreement of -32.7 to $64.1 \mathrm{~mm}^{2}$ (Figure $4 B, C, D, E, F, G, H)$ and -0.9 to $2.2 \mathrm{~mm}$ (Figure 5), respectively.

Figures 6 and 7 are examples for the measurements of carotid plaques on longitudinal and cross-sectional views of 2D ultrasound and 3D MR imaging.

\section{Reproducibility}

Table 3 summarizes the results of the reproducibility 

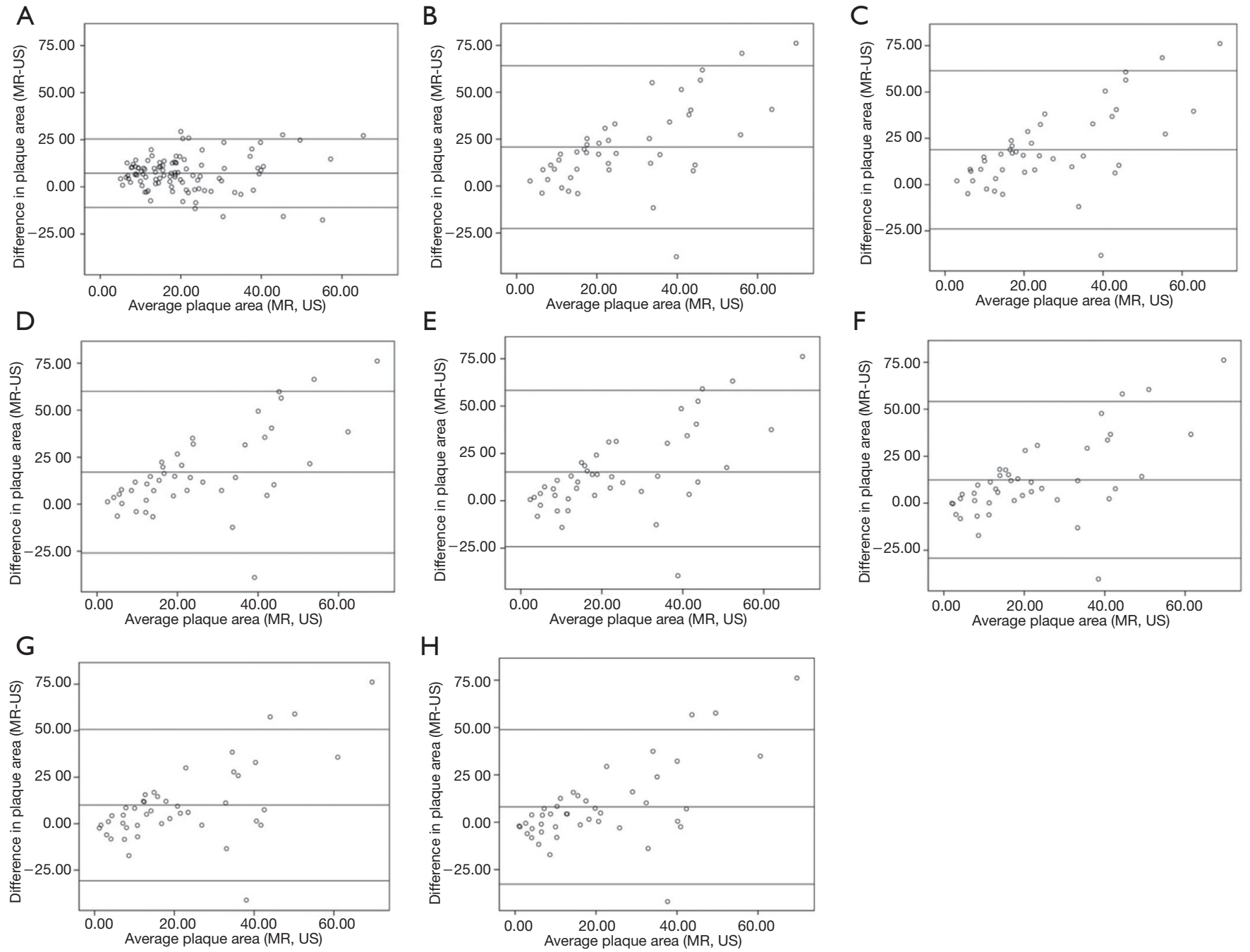

Figure 4 Bland-Altman analysis of plaque area measured by MR and ultrasound imaging on longitudinal views (A) and cross-sectional views (B,C,D,E,F,G,H). The plaque area on cross-sectional MR images was automatically calculated with different thresholds, which varied from $1.5 \mathrm{~mm}(\mathrm{~B})$ to $2.1 \mathrm{~mm}(\mathrm{H})$ with an interval of $0.1 \mathrm{~mm}$.

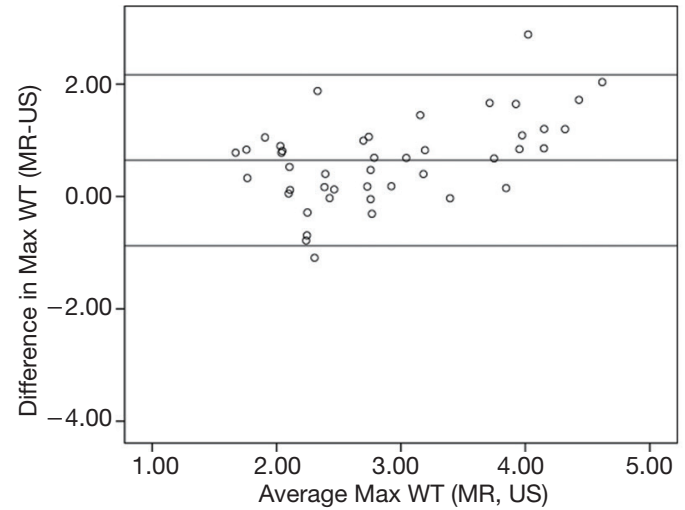

Figure 5 Bland-Altman analysis of Max WT measured by MR and ultrasound imaging on cross-sectional views. study. In measuring carotid Max WT, plaque length, and plaque area on longitudinal ultrasound images, the ICCs for inter-observer and intra-observer agreement ranged from 0.961 to 0.990 and from 0.981 to 0.997 , respectively. In measuring carotid Max WT and plaque area on crosssectional ultrasound images, the ICCs for inter-observer and intra-observer agreement were 0.894 and 0.937 , and 0.950 to 0.983 , respectively. In measuring carotid Max WT, plaque length, and plaque area on longitudinal MR images, the ICCs for inter-observer and intra-observer agreement ranged from 0.886 to 0.965 and from 0.927 to 0.982 , respectively. 
Table 2 Plaque area on MR using different wall thickness threshold and its association with plaque area on ultrasound

\begin{tabular}{lllr}
\hline Wall thickness threshold, $\mathrm{mm}$ & Plaque area, $\mathrm{mm}^{2}$ & \multicolumn{2}{c}{ Correlation with ultrasound } \\
\cline { 3 - 4 } & & $\mathrm{r}$ & $<$ \\
1.5 & $37.6 \pm 25.0$ & 0.603 & $<.001$ \\
1.6 & $35.6 \pm 24.9$ & 0.622 & $<0.001$ \\
1.8 & $33.9 \pm 25.3$ & 0.646 & $<.001$ \\
1.9 & $32.0 \pm 25.5$ & 0.647 & $<0.001$ \\
2.0 & $29.2 \pm 24.9$ & 0.660 & $<0.001$ \\
2.1 & $26.8 \pm 24.4$ & 0.687 & $<0.001$ \\
\hline
\end{tabular}

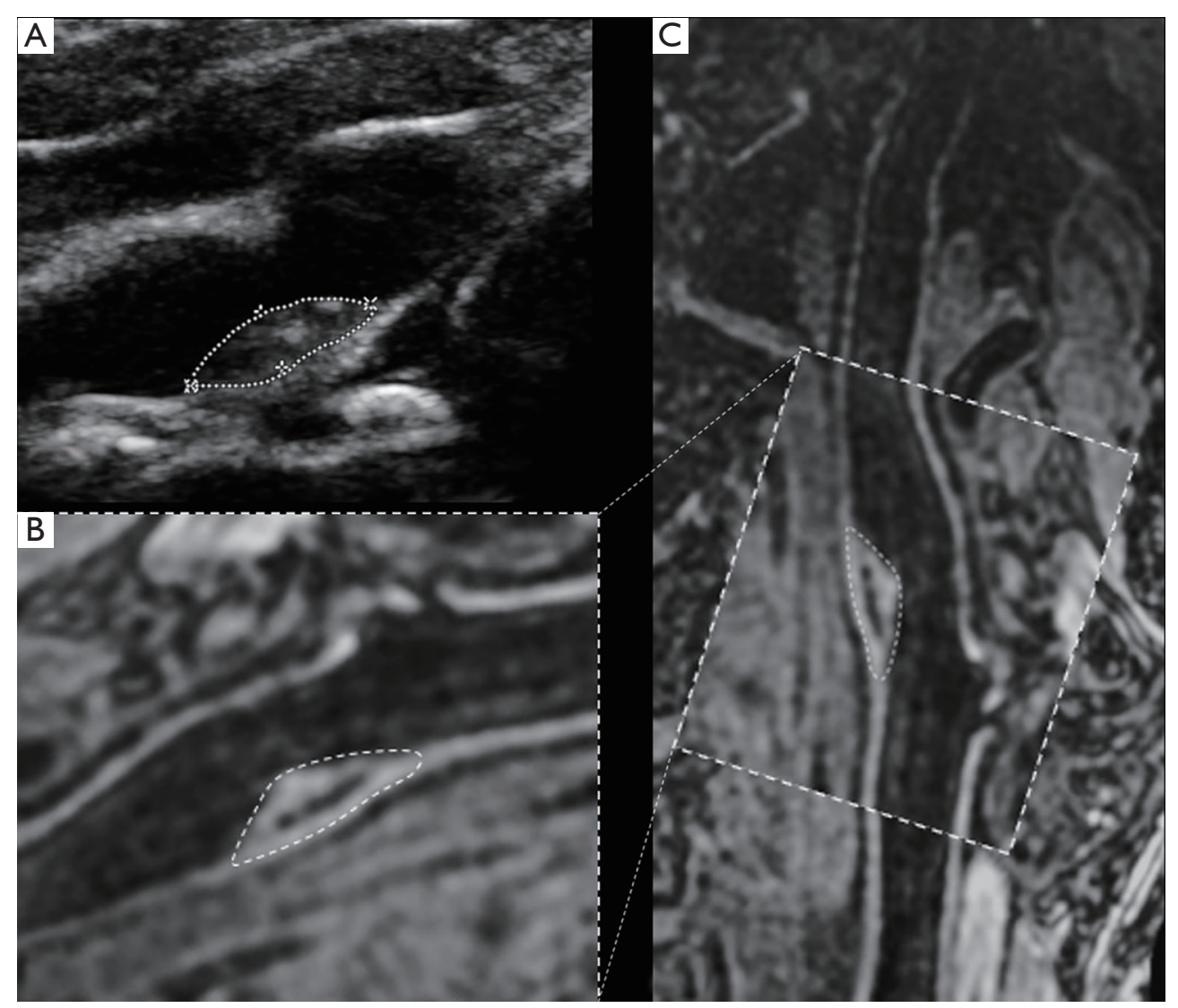

Figure 6 The measurements of carotid plaques on longitudinal views of both 2D ultrasound imaging (A) and 3D MR vessel wall imaging (B,C). The Max WT, plaque length, and plaque area of carotid atherosclerotic plaque (white circle) on 2D ultrasound and 3D MR imaging were as follows: 3.1 vs. $3.6 \mathrm{~mm}, 10.1$ vs. $10.5 \mathrm{~mm}$, and 22.4 vs. $26.0 \mathrm{~mm}^{2}$, respectively.

\section{Discussion}

In this study, we compared the Max WT, plaque length, and plaque area in carotid arteries and investigated their relationship between 3D MR and ultrasound imaging on longitudinal and cross-sectional views. We found that Max WT and plaque area measured by $3 \mathrm{D} M R$ imaging were significantly higher than those measured by ultrasound 

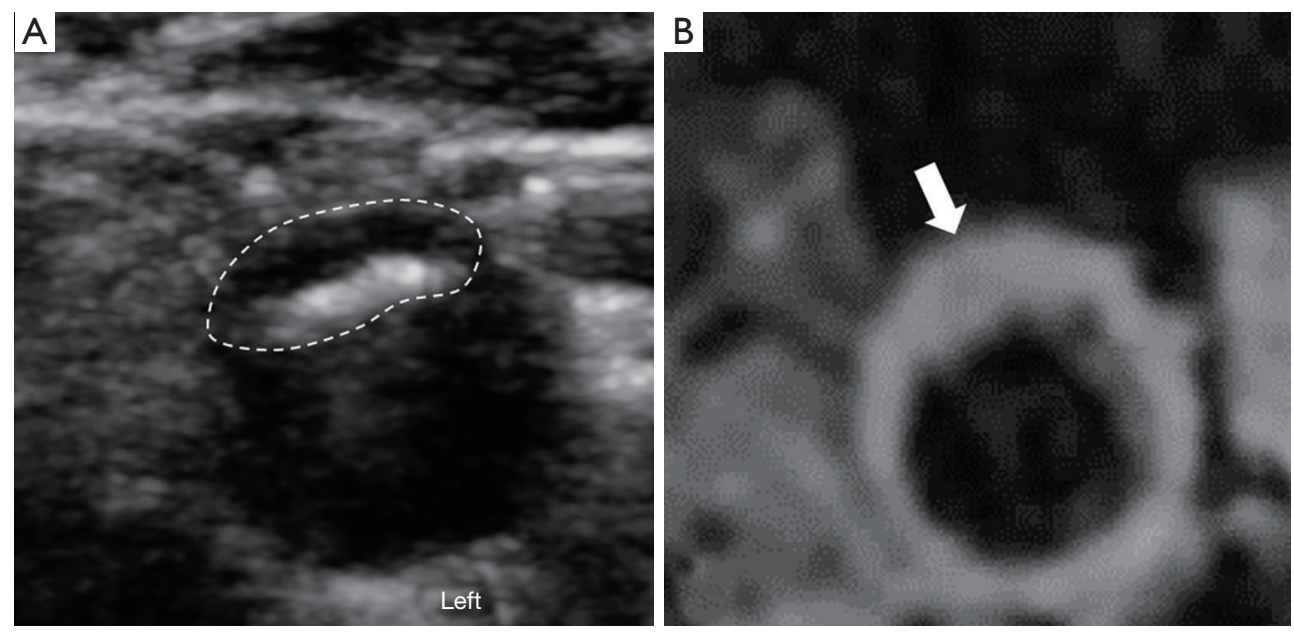

Figure 7 The measurements of carotid plaques on cross-sectional views of both 2D ultrasound imaging (A) and 3D MR vessel wall imaging (B, the same slice of Figure 1). The plaque area measured by 3D MR imaging with a different threshold of plaque wall thickness (1.5 to $2.1 \mathrm{~mm}$ ) ranged from 33.5 to $23.5 \mathrm{~mm}^{2}$. The Max WT and plaque area of carotid atherosclerotic plaque on 2D ultrasound (white circle) and 3D MR imaging (white arrow) were 2.6 vs. $2.8 \mathrm{~mm}$ and 16.1 vs. $23.5 \mathrm{~mm}^{2}$, respectively.

Table 3 Reproducibility of quantitatively measuring plaque size on ultrasound and MR images

\begin{tabular}{lcc}
\hline Imaging measurements & Inter-observer agreement, ICC $(95 \% \mathrm{Cl})$ & Intra-observer agreement, ICC $(95 \% \mathrm{Cl})$ \\
\hline Longitudinal ultrasound images & $0.961(0.852-0.990)$ & $0.981(0.925-0.995)$ \\
Max WT & $0.990(0.962-0.998)$ & $0.997(0.990-0.999)$ \\
Plaque length & $0.986(0.945-0.997)$ & $0.992(0.967-0.998)$ \\
Plaque area & & $0.950(0.814-0.987)$ \\
Cross-sectional ultrasound images & $0.894(0.631-0.973)$ & $0.983(0.932-0.996)$ \\
Max WT & $0.937(0.767-0.984)$ & $0.970(0.884-0.993)$ \\
Plaque area & & $0.927(0.734-0.981)$ \\
Longitudinal MR images & $0.886(0.608-0.970)$ & $0.982(0.928-0.995)$ \\
Max WT & $0.891(0.622-0.972)$ & $0.965(0.865-0.991)$ \\
Plaque length & & \\
Plaque area & & \\
\hline
\end{tabular}

imaging on both longitudinal and cross-sectional views. Moderate to strong correlations were found between MR and ultrasound imaging in measuring Max WT, plaque length, and plaque area.

In the present study, the Max WT and plaque area measured by MR imaging were more significant than those measured by ultrasound imaging, and the measurement of plaque length was similar between these two imaging modalities. The differences in plaque size between MR and ultrasound imaging might be due to the methodology of measurement. On 3D MR imaging, the wall thickness and plaque area were derived from the whole layers of the wall from the intima to adventitia. In contrast, ultrasound imaging can only confidently delineate and measure the thickness of intima and media in carotid arteries. Some investigators proposed measuring the extra-media thickness, but it not only consists of adventitia but also perivascular fat, interstitial tissue, and the venous wall (26). 
As such, the extra-media thickness is less often measured by a sonographer in clinical settings. In our study, the plaque size measurements on ultrasound imaging did not include adventitia and perivascular tissues. In histology, the adventitia accounts for $33 \%$ of whole wall thickness in healthy carotid arteries, and the mean thickness of adventitia was $0.35 \pm 0.16 \mathrm{~mm}$ after adjusted for the shrinkage artifact (27). In the present study, the Max WT measured by MR imaging was 0.5 and $0.6 \mathrm{~mm}$ greater than that measured by ultrasound imaging on longitudinal and cross-sectional views, respectively. This overestimation might be caused by the low resolution of MR imaging. Duivenvoorden et al. demonstrated that the measurements of wall thickness on MR images decreased with resolution increased from 0.65 to $0.2 \mathrm{~mm}$, and the in-plane resolution might be a reason for the difference in measurements between MR and ultrasound imaging. In our study, the resolution of MR imaging was $0.8 \mathrm{~mm}$ isotropic, which might introduce partial volume effects and lead to the overestimation of Max WT. The pulse of arteries may also blur the boundaries of the wall during MR imaging without electrocardiogramgating, which may also lead to the overestimation of the thickness of the arterial wall. These influence factors may also contribute to the more extensive measurements of plaque area on MR imaging as compared to ultrasound imaging.

In the present study, the semi-automatic measurement algorithm of the carotid plaque area was proposed on the cross-sectional MR images. This study is one of the first to investigate the plaque area measured on MR images. In our study, the thresholds for the automatic algorithm of carotid plaque area on cross-sectional MR images were set as 1.5 to $2.1 \mathrm{~mm}$ with an interval of $0.1 \mathrm{~mm}$. This is because the intima-media thickness of carotid plaque on ultrasound imaging was usually thicker than $1.5 \mathrm{~mm}$ (28) and the Max WT measured on cross-sectional MR images was $0.6 \mathrm{~mm}$ greater than that measured on cross-sectional ultrasound images. Earlier ultrasound studies showed the carotid plaque area was a strong predictor for ischemic stroke (7). However, in carotid atherosclerosis MR imaging studies, the plaque area has received less attention than the Max WT and the area of each plaque component.

The carotid plaque area ranged from 24.9 to $37.6 \mathrm{~mm}^{2}$ among different thresholds of wall thickness on crosssectional MR images in our study. An earlier correlation study between high-resolution MR and histopathology reported that the maximum carotid wall area on $M R$ imaging ranged from 46.4 to $54.2 \mathrm{~mm}^{2}$ (29). Furthermore, our study also showed that the carotid plaque area measured by MR images was around 1.43 -fold higher than that measured by ultrasound images on longitudinal views. When the threshold was set as $2.1 \mathrm{~mm}$ for wall thickness, the plaque area measured by MR imaging was 1.48 -fold higher than that measured by ultrasound imaging on crosssectional views, which is closest to the measurements on the longitudinal views among all thresholds. The threshold of $2.1 \mathrm{~mm}$ yielded the strongest correlation of plaque area between MR and ultrasound imaging on cross-sectional views. In clinical settings, this threshold can be used to guide the automatic identification of carotid plaques on MR images. Considering the low variability of $M R$ imaging, $M R$ imaging might possess higher sensitivity in finding carotid plaques than ultrasound imaging. However, it should be noticed that the workflows for measuring wall thickness and plaque area on MR and ultrasound imaging were different. The measurements of plaque thickness, plaque length, and plaque area on ultrasound images from both longitudinal and cross-sectional views were conducted by an experienced sonographer. The measurement of Max WT on MR images from a cross-sectional view was acquired by two experienced radiologists with 3D CASCADE software, and the plaque area on MR images from a cross-sectional view was measured using a semi-automatic measurement algorithm based on the wall thickness. The measurements of Max WT, plaque length, and plaque area on MR images from a longitudinal view were conducted by two experienced radiologists. These different workflows might also influence the differences in quantitative measurements of carotid plaque between ultrasound and MR images. In addition, the correlation coefficients of Max WT and plaque area measured by 2D ultrasound and 3D MR images from a cross-sectional view were slightly higher than those from a longitudinal view. In contrast, Bland-Altman analyses showed that the bias and the limits of agreement of Max WT and plaque area measured by 2D ultrasound and 3D MR images from a longitudinal view were smaller than those from a cross-sectional view. These might be caused by the different workflows on longitudinal and cross-sectional analyses.

This study has several limitations. First, the sample size for the analysis on cross-sectional views was smaller than that on longitudinal views because cross-sectional ultrasound scan was not routine. Larger sample sizes for the comparison between ultrasound and MR on cross-sectional views are called for in the future. Second, the clinical application of our threshold on MR images in checking the 
progression of plaque has not been proved and follow-up studies are called for in the future. Third, the resolution of 3D MR imaging was $0.8 \mathrm{~mm}$ isotropic, which is larger than the standard adventitia thickness and could cause a partial volume effect. The difference in carotid plaque measurements between MR and ultrasound imaging might be attributed to both the adventitia thickness and the partial volume effect. MR imaging with higher spatial resolution might improve the accuracy of quantitative measurements. Finally, the plaque size measured by $2 \mathrm{D}$ ultrasound is heavily dependent on the orientation of the scan by the sonographer. Recently, 3D ultrasound imaging has been used to assess carotid plaques, which may enable more accurate quantitative assessments $(30,31)$.

The quantitative measurements of carotid plaques using 3D MR and 2D ultrasound imaging are significantly correlated. The plaque area and Max WT measured by 3D MR imaging are more extensive than these parameters measured by $2 \mathrm{D}$ ultrasound imaging, which might be explained by both the resolution of MR and the workflow of measurements.

\section{Acknowledgments}

Funding: This work was supported by the grants of the National Natural Science Foundation of China (81771825), Beijing Municipal Science and Technology Project (D171100003017003, D131100002313002) and Ministry of Science and Technology of People's Republic of China (2017YFC1307904).

\section{Footnote}

Conflicts of Interest: All authors have completed the ICMJE uniform disclosure form (available at http://dx.doi. org/10.21037/qims-19-818). The authors have no conflicts of interest to declare.

Ethical Statement: The study protocol was supported by the local institutional review board, and all participants completed a written informed consent form.

Open Access Statement: This is an Open Access article distributed in accordance with the Creative Commons Attribution-NonCommercial-NoDerivs 4.0 International License (CC BY-NC-ND 4.0), which permits the noncommercial replication and distribution of the article with the strict proviso that no changes or edits are made and the original work is properly cited (including links to both the formal publication through the relevant DOI and the license). See: https://creativecommons.org/licenses/by-nc-nd/4.0/.

\section{References}

1. Parmar JP, Rogers WJ, Mugler JR, Mugler JP 3rd, Baskurt E, Altes TA, Nandalur KR, Stukenborg GJ, Phillips CD, Hagspiel KD, Matsumoto AH, Dake MD, Kramer CM. Magnetic resonance imaging of carotid atherosclerotic plaque in clinically suspected acute transient ischemic attack and acute ischemic stroke. Circulation 2010;122:2031-8.

2. Gupta A, Baradaran H, Schweitzer AD, Kamel H, Pandya A, Delgado D, Dunning A, Mushlin AI, Sanelli PC. Carotid plaque MRI and stroke risk: a systematic review and meta-analysis. Stroke 2013;44:3071-7.

3. Zavodni AE, Wasserman BA, McClelland RL, Gomes AS, Folsom AR, Polak JF, Lima JA, Bluemke DA. Carotid artery plaque morphology and composition in relation to incident cardiovascular events: the Multi-Ethnic Study of Atherosclerosis (MESA). Radiology 2014;271:381-9.

4. Zhao X, Underhill HR, Zhao Q, Cai J, Li F, Oikawa M, Dong L, Ota H, Hatsukami TS, Chu B, Yuan C. Discriminating carotid atherosclerotic lesion severity by luminal stenosis and plaque burden: a comparison utilizing high-resolution magnetic resonance imaging at 3.0 Tesla. Stroke 2011;42:347-53.

5. Spence JD, Eliasziw M, DiCicco M, Hackam DG, Galil R, Lohmann T. Carotid plaque area: a tool for targeting and evaluating vascular preventive therapy. Stroke 2002;33:2916-22.

6. Zhao H, Zhao X, Liu X, Cao Y, Hippe DS, Sun J, Li F, $\mathrm{Xu}$ J, Yuan C. Association of carotid atherosclerotic plaque features with acute ischemic stroke: a magnetic resonance imaging study. Eur J Radiol 2013;82:e465-70.

7. Mathiesen EB, Johnsen SH, Wilsgaard T, Bønaa KH, Løchen ML, Njølstad I. Carotid plaque area and intimamedia thickness in prediction of first-ever ischemic stroke: a 10-year follow-up of 6584 men and women: the Tromsø Study. Stroke 2011;42:972-8.

8. Lorenz MW, Markus HS, Bots ML, Rosvall M, Sitzer M. Prediction of clinical cardiovascular events with carotid intima-media thickness: a systematic review and metaanalysis. Circulation 2007;115:459-67.

9. Dwyer JH, Sun P, Kwong-Fu H, Dwyer KM, Selzer RH. Automated intima-media thickness: the Los Angeles Atherosclerosis Study. Ultrasound Med Biol 
1998;24:981-7.

10. Li F, Yarnykh VL, Hatsukami TS, Chu B, Balu N, Wang J, Underhill HR, Zhao X, Smith R, Yuan C. Scanrescan reproducibility of carotid atherosclerotic plaque morphology and tissue composition measurements using multicontrast MRI at 3T. J Magn Reson Imaging 2010;31:168-76.

11. Kang X, Polissar NL, Han C, Lin E, Yuan C. Analysis of the measurement precision of arterial lumen and wall areas using high-resolution MRI. Magn Reson Med 2000;44:968-72.

12. Sun J, Zhao XQ, Balu N, Hippe DS, Hatsukami TS, Isquith DA, Yamada K, Neradilek MB, Cantón G, Xue Y, Fleg JL, Desvigne-Nickens P, Klimas MT, Padley RJ, Vassileva MT, Wyman BT, Yuan C. Carotid magnetic resonance imaging for monitoring atherosclerotic plaque progression: a multicenter reproducibility study. Int J Cardiovasc Imaging 2015;31:95-103.

13. Schwarz F, Strobl FF, Cyran CC, Helck AD, Hartmann M, Schindler A, Nikolaou K, Reiser MF, Saam T. Reproducibility and differentiation of cervical arteriopathies using in vivo high-resolution black-blood MRI at 3 T. Neuroradiology 2016;58:569-76.

14. van Wijk DF, Strang AC, Duivenvoorden R, Enklaar DJ, van der Geest RJ, Kastelein JJ, de Groot E, Stroes ES, Nederveen AJ. Increasing spatial resolution of 3T MRI scanning improves reproducibility of carotid arterial wall dimension measurements. MAGMA 2014;27:219-26.

15. Li D, Dai W, Cai Y, Han Y, Yao G, Chen H, Yuan C, Xiao $\mathrm{L}$, Zhao X. Atherosclerosis in stroke-related vascular beds and stroke risk: A 3-D MR vessel wall imaging study. Ann Clin Transl Neurol 2018;5:1599-610.

16. Saba L, Yuan C, Hatsukami TS, Balu N, Qiao Y, DeMarco JK, Saam T, Moody AR, Li D, Matouk CC, Johnson MH, Jäger HR, Mossa-Basha M, Kooi ME, Fan Z, Saloner D, Wintermark M, Mikulis DJ, Wasserman BA; Vessel Wall Imaging Study Group of the American Society of Neuroradiology. Carotid Artery Wall Imaging: Perspective and Guidelines from the ASNR Vessel Wall Imaging Study Group and Expert Consensus Recommendations of the American Society of Neuroradiology. AJNR Am J Neuroradiol 2018;39:E9-E31.

17. Zhou Z, Li R, Zhao X, He L, Wang X, Wang J, Balu N, Yuan C. Evaluation of 3D multi-contrast joint intra- and extracranial vessel wall cardiovascular magnetic resonance. J Cardiovasc Magn Reson 2015;17:41.

18. Fan Z, Yu W, Xie Y, Dong L, Yang L, Wang Z, Conte AH, Bi X, An J, Zhang T, Laub G, Shah PK, Zhang Z, Li D.
Multi-contrast atherosclerosis characterization (MATCH) of carotid plaque with a single 5-min scan: technical development and clinical feasibility. J Cardiovasc Magn Reson 2014;16:53.

19. Li L, Chai JT, Biasiolli L, Robson MD, Choudhury RP, Handa AI, Near J, Jezzard P. Black-blood multicontrast imaging of carotid arteries with DANTE-prepared 2D and 3D MR imaging. Radiology 2014;273:560-9.

20. Duivenvoorden R, de Groot E, Elsen BM, Laméris JS, van der Geest RJ, Stroes ES, Kastelein JJ, Nederveen AJ. In vivo quantification of carotid artery wall dimensions: 3.0-Tesla MRI versus B-mode ultrasound imaging. Circ Cardiovasc Imaging 2009;2:235-42.

21. Duivenvoorden R, de Groot E, Afzali H, Vanbavel ET, de Boer OJ, Laméris JS, Fayad ZA, Stroes ES, Kastelein JJ, Nederveen AJ. Comparison of in vivo carotid 3.0-T magnetic resonance to $\mathrm{B}$-mode ultrasound imaging and histology in a porcine model. JACC Cardiovasc Imaging 2009;2:744-50.

22. Underhill HR, Kerwin WS, Hatsukami TS, Yuan C. Automated measurement of mean wall thickness in the common carotid artery by MRI: a comparison to intimamedia thickness by B-mode ultrasound. J Magn Reson Imaging 2006;24:379-87.

23. Touboul PJ, Hennerici MG, Meairs S, Adams H, Amarenco P, Desvarieux M, Ebrahim S, Fatar M, Hernandez Hernandez R, Kownator S, Prati P, Rundek T, Taylor A, Bornstein N, Csiba L, Vicaut E, Woo KS, Zannad F; Advisory Board of the 3rd Watching the Risk Symposium 2004, 13th European Stroke Conference. Mannheim intima-media thickness consensus. Cerebrovasc Dis 2004; 18:346-349.

24. Cai Y, He L, Yuan C, Chen H, Zhang Q, Li R, Li C, Zhao X. Atherosclerotic plaque features and distribution in bilateral carotid arteries of asymptomatic elderly population: A 3D multicontrast MR vessel wall imaging study. Eur J Radiol 2017;96:6-11.

25. Yuan C, Mitsumori LM, Ferguson MS, Polissar NL, Echelard D, Ortiz G, Small R, Davies JW, Kerwin WS, Hatsukami TS. In vivo accuracy of multispectral magnetic resonance imaging for identifying lipid-rich necrotic cores and intraplaque hemorrhage in advanced human carotid plaques. Circulation 2001;104:2051-6.

26. Skilton MR, Boussel L, Bonnet F, Bernard S, Douek PC, Moulin P, Serusclat A. Carotid intima-media and adventitial thickening: comparison of new and established ultrasound and magnetic resonance imaging techniques. Atherosclerosis 2011;215:405-10. 
27. Gamble G, Beaumont B, Smith H, et al. B-mode ultrasound images of the carotid artery wall: correlation of ultrasound with histological measurements. Atherosclerosis 1993;102:163-73.

28. Touboul PJ, Hennerici MG, Meairs S, Adams H, Amarenco P, Bornstein N, Csiba L, Desvarieux M, Ebrahim S, Hernandez Hernandez R, Jaff M, Kownator S, Naqvi T, Prati P, Rundek T, Sitzer M, Schminke U, Tardif JC, Taylor A, Vicaut E, Woo KS. Mannheim carotid intima-media thickness and plaque consensus (2004-20062011). An update on behalf of the advisory board of the $3 \mathrm{rd}, 4$ th and 5 th watching the risk symposia, at the 13 th, 15th and 20th European Stroke Conferences, Mannheim, Germany, 2004, Brussels, Belgium, 2006, and Hamburg, Germany, 2011. Cerebrovasc Dis 2012;34:290-6.

Cite this article as: Qiao H, Cai Y, Huang M, Liu Y, Zhang Q, Huang L, Chen H, Yuan C, Zhao X. Quantitative assessment of carotid artery atherosclerosis by three-dimensional magnetic resonance and two-dimensional ultrasound imaging: a comparison study. Quant Imaging Med Surg 2020;10(5):10211032. doi: 10.21037/qims-19-818
29. Xia Z, Yang H, Yuan X, Wang J, Zhang S, Zhang L, Qu Y, Chen J, Jiao L, Wang LX, Du Y. High-resolution magnetic resonance imaging of carotid atherosclerotic plaques - a correlation study with histopathology. Vasa 2017;46:283-90.

30. Chiu B, Egger M, Spence JD, Parraga G, Fenster A. Quantification of carotid vessel wall and plaque thickness change using 3D ultrasound images. Med Phys 2008;35:3691-710.

31. Yamaguchi M, Sasaki M, Ohba H, Mori K, Narumi S, Katsura N, Ohura K, Kudo K, Terayama Y. Quantitative assessment of changes in carotid plaques during cilostazol administration using three-dimensional ultrasonography and non-gated magnetic resonance plaque imaging. Neuroradiology 2012;54:939-45. 